\title{
An Adaptive Location-Aware MAC Protocol for Multichannel Multihop Ad-Hoc Networks
}

\author{
Zi-Tsan Chou ${ }^{1,2}$, Ching-Chi Hsu ${ }^{1,3}$, and Ferng-Ching Lin ${ }^{1,2}$ \\ 1 Department of Computer Science and Information Engineering \\ National Taiwan University, Taipei, 106, Taiwan \\ \{d5526005, cchsu, fc_lin\}@csie.ntu.edu.tw \\ 2 Institute for Information Industry, Taipei, 106, Taiwan \\ \{ztchou, fclin\}@iii.org.tw \\ 3 Kai Nan University, Tauyan, Taiwan
}

\begin{abstract}
In a multihop MANET (mobile ad-hoc network), reliable broadcast support at the MAC layer will be of great benefit to the routing function, multicasting applications, cluster maintenance, and realtime systems. In this paper, we propose a new hybrid MAC protocol, called the adaptive location-aware broadcast (ALAB) protocol, for linklevel broadcast support in multichannel systems. ALAB is scalable and mobility-transparent since it does not require any link state information. Above all, in ALAB, both deadlock and hidden terminal problems are completely solved. In principle, ALAB tries to combine both of the advantages of the allocation- and contention-based protocols and overcomes their individual drawbacks. At high traffic or density, ALAB outperforms the pure TDMA because of spatial reuse and dynamic slot management. At low traffic or density, ALAB outperforms the pure CSMA/CA because of its embedded stable tree-splitting algorithms. In addition, ALAB provides deterministic access delay bounds from its base TDMA allocation protocol. Simulation results do confirm the advantage of our scheme over other MAC protocols, such as IEEE 802.11, ADAPT, and ABROAD, even under the fixed-total-bandwidth model.
\end{abstract}

\section{Introduction}

With the revolutionary advances of wireless technology, the applications of the MANET (mobile ad-hoc network) are getting more and more important, especially in the emergency, military, and outdoor business environments, in which instant fixed infrastructure or centralized administration is difficult or too expensive to establish. In the MANET, pair of nodes communicates by sending packets either over a direct wireless link or through a sequences of wireless links including some intermediate nodes. Due to the broadcast nature of the radio transmission medium and the rapidly dynamic topology changes in the MANET, every algorithms and protocols developed on it will face great challenges. In this paper, we are specially interested in a medium access control (MAC) protocol for multihop ad-hoc networks with multiple frequency channels. 
A MAC protocol is to address how to allocate the multiaccess medium and resolve potential contention/collision among various nodes. MAC protocols proposed so far can be approximately classified into two categories [5]. One is allocation-based protocols, and the other is contention-based protocols. Deterministic allocation-based protocols, such as TDMA and its variants [9, are primarily designed to support bounded delay topology-independent transmissions by scheduled slot assignments. Nevertheless, these protocols are insensitive to variations in network loads or node connectivity. Although dynamic topologydependent TDMA-based transmission scheduling protocols [13] can adjust themselves to node connectivity, they are not suitable for highly mobility environments due to heavy loads on updated link state information maintenance. As to the contention-based protocols, such as CSMA/CA and it variants [14, they are primarily designed to support asynchronous transmissions and burst traffic. However, CSMA/CA is inherently unstable [7]. Because of this reason, the CARMA protocol based on the deterministic tree-splitting algorithm [7] was proposed. In CARMA, in order to maintain a consistent channel view for all nodes in a multihop wireless network, a base station should be set up to govern this task. Hence it is not suitable for the large-scale MANET.

Most previous works on MAC protocols including IEEE 802.11, ADAPT 4, CARMA [7, and GRID [14] are designed to support only reliable unicast transmission. As indicated in 5,811], support for reliable broadcast at the MAC layer will be of great benefit to the routing function, multicasting applications, cluster maintenance, and real-time systems. Clearly, a single reliable broadcast can be implemented by sending one or more reliable unicast messages. However, this approach is not scalable since the time to complete a broadcast increases with the number of neighbors. Besides, MAC protocols typically do not maintain link state information [5]. Recently, several MAC protocols for broadcast support have been proposed, including ABROAD [5], TPMA [8], RBRP [1], CATA 13], and FPRP [15]. All of them depend on the collision detection capacity. In TPMA and RBRP, nodes with bad luck in their elimination phase or reservation request phase may lead to starvation. To make matters worse, all of these protocols may lead to deadlocks. A deadlock [11] is said to occur if two conflicting broadcasts are scheduled in the same slot and the senders do not realize this conflict. We also notice that all the above-mentioned protocols have focused only on single channel systems. From many literatures [914], we know that a multichannel system outperforms a single channel system in many aspects, including throughput, reliability, bandwidth utilization, network scalability, synchronization implementation, and QoS support.

The authors in [5] developed a novel hybrid MAC protocol, called ABROAD, for reliable broadcast in single channel MANETs. Importantly, they try to combine both of the advantages of the allocation- and contention-based protocols and overcomes their individual drawbacks. Thus, ABROAD can dynamically self-adjust its behavior according to the prevailing network conditions [45]. Following their hybrid approach, but with a whole different design strategy, we propose a new multichannel MAC protocol based on the tree-splitting algo- 
rithms for link-level broadcast support in multihop ad-hoc networks. We call the resulting distributed protocol "Adaptive Location-Aware Broadcast" (ALAB) protocol. Since a MANET should operate in a physical area, it is very natural to exploit location information in such an environment 14. In addition, via a GPS (Global Positioning Systems), every node can get absolute timing and location information; thus synchronization becomes easy 8,1112. The advantages of the ALAB protocol are as follows. (i) ALAB supports reliable unicast, multicast, and broadcast transmission services in an integrated manner. That is, unicast and multicast packets are considered as special cases of broadcast packets. (ii) ALAB is scalable and mobility-transparent since it does not require any link state information. Moreover, both the time to broadcast a packet and the number of channels required for the MANET are independent of the network topology. (iii) In ALAB, no hidden or exposed terminal problems will exist. Therefore, our design does not need any handshake process such as RTS/CTS or RTS/CR/RA [8]. (iv) Like ABROAD, ALAB also provides bounded access delay from its base TDMA allocation protocol. Furthermore, ALAB is stable and adapts well to any traffic and network topologies. (v) In ALAB, the deadlock and starvation problems are completely eliminated. (vi) Under the severe traffic load and node density conditions, ALAB delivers superior performance than ABROAD, which outperforms TDMA, IEEE 802.11, and ADAPT [45], even under the fixed-total-bandwidth model.

\section{The ALAB Protocol}

\subsection{Model and Assumptions}

A multihop mobile radio network used to pass messages containing data and control information can be modelled as an undirected graph $G=(\mathcal{V}, \mathcal{E})$ in which $\mathcal{V}(|\mathcal{V}|=N)$ is the set of mobile hosts and there is an edge $(u, v) \in \mathcal{E}$ if and only if $u$ and $v$ can mutually receive each other's transmissions. In this case, we say that $u$ and $v$ are neighbors. Note that the edge set may vary over time because of nodal mobility. We can assign each node $v$ in the network a unique identifer $(I D)$ by a number in $\aleph=\{0,1, \ldots, N-1\}$, where $|\aleph|=N$. In this paper, all logarithms are assumed to be base 2. Given an integer $v \in \aleph$, let $\operatorname{Binary}(v)=\left(v_{1} v_{2} \ldots v_{k-1} v_{k}\right)$ denote its binary string, where $k=\lceil\log N\rceil$. Thus, every integer in $\aleph$ can be represented by a unique binary $k$-tuples $\left(v_{1} v_{2} \ldots v_{k-1} v_{k}\right)$, where $v_{i} \in\{0,1\}$. In addition, each channel is uniquely assigned by a number in $\mathcal{C}=\{0,1, \ldots, \rho-1\}$, where $1 \leq \rho<N$.

Within a TDMA network, the time axis is divided into units called (transmission) frames, and each frame is composed of time slots. Each slot in turn comprises mini-slots. Nodes in the network are assumed to be synchronized and that the frame length is the same for each node. Each mobile radio host in a multichannel network is equipped with the transceivers (a single transmitter and multiple receivers). Depending on the ability of the transceivers, each node can communicate with others either in the full-duplex mode or in the half-duplex mode. In the half-duplex mode, each host cannot transmit and receive at the 


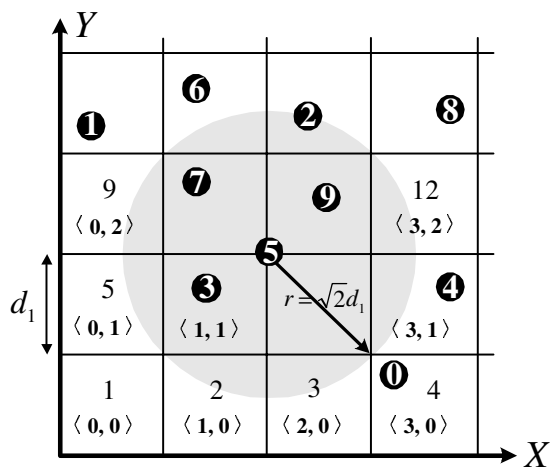

Fig. 1. Ten mobile hosts are dispersed randomly over the $2 \mathrm{D}$ geographic region. The integer within in the grid is the channel number, while the integer pairs are the grid coordinate. The center part of the geographic area shows the relation between $r$ and $d$.

same time [3]. In the full-duplex mode, each host can transmit only one packet on one channel but receive multiple packets on all channels simultaneously [9]. Throughout this paper, we assume every node works in the full-duplex mode. On the same channel, two types of communication collisions will arise [9]. The primary collision occurs when a node transmitting in a given mini-slot is receiving in the same mini-slot on the same channel. This also implies the converse: a receiving node cannot be transmitting on the same channel at the same time. The secondary collision occurs when node receives more than one packet in a mini-slot on the same channel. In both cases, all packets are rendered useless. To this end, we assume that if more than one node is transmitting on the same channel such that the packets overlap in time, then collision occurs on that channel. On the other hand, simultaneous reception of packets on other channels is not affected [39]. In this paper, we also assume that a node is capable of determining the current status of a single radio channel [12]. That is, at the end of a mini-slot, each node can obtain feedback from the receiver specifying whether the status of a radio channel is (i) NULL: no transmission on the channel, (ii) SINGLE: exactly one transmission on the channel, or (iii) COLLISION: two or more transmissions on the channel.

The basic idea behind the ALAB protocol is very simple; in brief, we just imitate the organization of cellular/cluster networks. This approach is widely adopted in many issues for the MANET 6 14]. Each node is assumed to know its own position by virtue of its GPS receiver but not the position of any other nodes in the network. In our model of the ad-hoc network, nodes are dispersed randomly over a pre-defined geographic region, which is partitioned into twodimensional logical grids as illustrated in Fig. 1. Each grid is a square of size $d \times d$. Let $r_{i}$ be the transmission range of node $i$. Determining the optimal values of $r_{i}$ and $d$ is not an easy task. In our design, we restrict $\sqrt{2} d \leq r_{i} \leq 2 d$. Let $\aleph_{\infty}=\{0,1,2,3, \ldots\}$. Grids are numbered $\langle x, y\rangle$ following the conventional $x y$ coordinate. Every node must know how to map its physical location $\left(x^{\prime}, y^{\prime}\right) \in$ 
TDMA frame

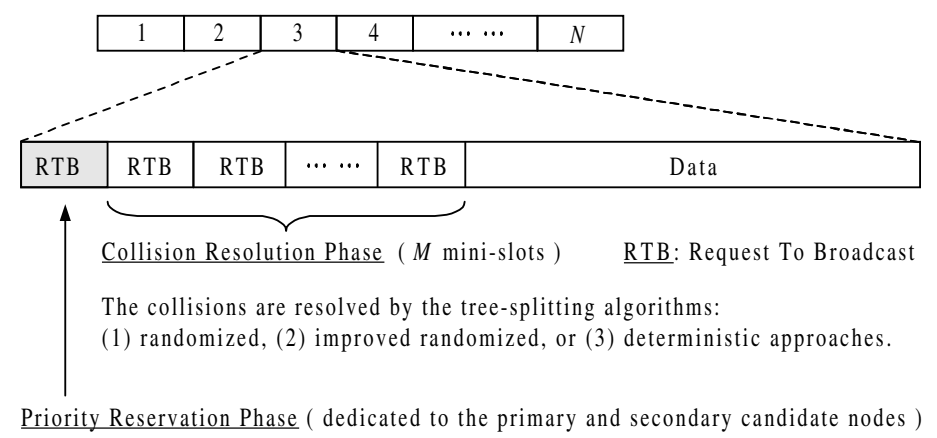

Fig. 2. The ALAB slot and frame structure.

$(\Re, \Re)$ to the corresponding grid coordinate $\langle x, y\rangle \in\left\langle\aleph_{\infty}, \aleph_{\infty}\right\rangle$. As illustrated in Fig. 1. we assign $\langle x, y\rangle=\left\langle\left\lfloor\frac{x^{\prime}}{d}\right\rfloor,\left\lfloor\frac{y^{\prime}}{d}\right\rfloor\right\rangle$. Besides, each grid is assigned a unique channel. When a node is located at a grid $\langle x, y\rangle$, it must use the channel assigned to the grid $\langle x, y\rangle$ for transmission. Given two different girds $\left\langle x_{1}, y_{1}\right\rangle$ and $\left\langle x_{2}, y_{2}\right\rangle$, if $\max \left\{\left|x_{1}-x_{2}\right|,\left|y_{1}-y_{2}\right|\right\} \leq 4$, then these two girds are called the interfering grids. The interfering grids are forbidden to be assigned the same channel to prevent co-channel interference in the packet transmission phase of ALAB. (We will explain it in the next subsection.) To attain this goal, we can simply apply the distance-4 coloring algorithms [14] to assign a channel for each grid. In the meantime, the frequency reuse should be maximized. Fig. 11 shows the possible channel assignments. Let $|\mathcal{G}|$ be the total number of grids over the geographic region. By a simple counting, the total number of channels required for the ALAB protocol is $\min \{25,|\mathcal{G}|\}$.

The main purposes of these restrictions are as follows. Nodes within the same grid form a single-hop cluster. In other words, all nodes within the same grid can hear the transmission of others. By the collision detection ability of the transceivers, all nodes within the same grid are able to maintain a consistent channel view. Due to the channel consistency in every grid, no deadlock or hidden terminal problems will exist.

\subsection{Protocol Description}

The ALAB protocol integrates a tree-splitting collision resolution protocol within each slot of a TDMA allocation protocol. Each node is assigned a transmission schedule (frame) consisting of $N$ slots. The slot and frame structure of the ALAB protocol, which is somewhat like the design of HYPERLAN [1] is shown in Fig. 2. The frame is divided into fixed-sized slots. Each slot is composed of three parts: a priority reservation phase and a collision resolution phase followed by a packet transmission phase. The first two phases are called the leader election phase. The priority reservation phase occupies the first mini-slot and the collision resolution phase consists of the next $M$ mini-slots. The final mini-slot is the 
packet transmission phase. In the priority reservation phase, only the predetermined primary and secondary candidate nodes have the chance to reserve the slot. However, when the first mini-slot remains unused, all active nodes contend to use it either by the randomized collision resolution algorithm or by the deterministic one. A node is said an active node if it has packets to send. Through the leader election phase, we guarantee that at most one active node will survive in a gird. The survival(s) gets the right of broadcast in the packet transmission phase. Recall that the transmission range is limited and simultaneous reception of packets on other channels is not affected. As a result, inter-grid communications via data packets are collision-free. In the following, we will focus the protocol description on a single grid, say $\langle x, y\rangle$. Before we describe the ALAB protocol in detail, we also make the following assumptions. (i) A node located in a grid $\langle x, y\rangle$ is assumed to continuously monitor the status of the channel assigned to $\langle x, y\rangle$. (ii) A node wishing to transmit a packet which may has arrived in the interim, it would wait until the beginning of the next slot. (iii) The channel introduces no errors, so control-packet collisions are the only source of errors. Nodes can perfectly detect such collisions immediately at the end of each minislot. (iv) Each mini-slot is designed to accommodate the packet transmission time and the guard time, which corresponds to the maximum differential propagation delay between any pair of nodes. Since the network are assumed to be synchronized, all active nodes enter the priority reservation phase synchronously.

1) Priority Reservation Phase: In slot $i$ of a frame, we let the node with the $I D=i-1 \bmod N$ be the primary candidate (PC for short) node and the node with the $I D=i-1+\left\lfloor\frac{N}{2}\right\rfloor \bmod N$ be the secondary candidate (SC for short) node. In our design, the priority of the $\mathrm{PC}$ node is higher than that of the $\mathrm{SC}$ node. At the beginning of the first mini-slot, only the PC and SC nodes are allowed to send RTB (Request-To-Broadcast) control packets with probability 1. At the end of the first mini-slot, if the status of the channel is COLLISION, then the PC node overwhelmingly wins the slot to broadcast a packet. If the status of the channel is SINGLE, all active nodes except the winner quit the contention at the remaining mini-slots, abandon the corresponding packet transmission minislot and wait for the next slot. Otherwise, all active nodes enter the collision resolution phase.

2.a) Randomized Collision Resolution Phase: At the beginning of the $i$ th mini-slot, where $2 \leq i \leq M+1$, all active nodes send RTBs with probability 1 . At the end of the $i$ th mini-slot, if the status of the channel is NULL, then the collision resolution period is over. The contending nodes involved in the COLLISION split randomly into two subsets by each flipping a coin. Those who obtain heads (with probability $p$ ) send an RTB in the next mini-slot; while those who obtain tails (with probability $1-p$ ) become inactive and wait for the next slot. This process keeps running until a SINGLE is reported or $i$ equals $M+1$, whichever comes first. The above-mentioned algorithm is similar to that of TPMA [8]. We can find that the collision resolution process stops immediately once a NULL occurs. One can make a further improvement, however. On condition that a NULL is sensed, all previous contenders are allowed to flip a coin 
again and those who obtain heads can send RTBs in the next mini-slot. Thus the collision resolution phase will never terminate in the NULL state before mini-slot $M+1$. It is worth noticing that nodes with bad luck in the randomized collision resolution phase will not starve because of the underlying TDMA protocol. The advantages of the randomized approach are that it achieves fairness naturally and a winner may arise quickly. A reasonable value of $M$ could be $1+\left\lceil\log \frac{N}{|\mathcal{G}|}\right\rceil$.

2.b) Deterministic Collision Resolution Phase: Our deterministic collision resolution algorithm is similar to that in [2]. We assume that every node keeps an integer variable temporary_ID used for the collision resolution phase. Initially, temporary_ID $=I D$. We let $M=1+\lceil\log N\rceil$ and $\left(b_{1} b_{2}, \cdots, b_{k}\right)$ be the binary representation of any given node temporary_ID, where $k=\lceil\log N\rceil$. At the beginning of the second mini-slot, all active nodes send RTB packets. If the status of the channel is NULL, then the collision resolution period is over. If a COLLISION occurs, all active nodes with $b_{1}=0$ send RTB packets in the next mini-slot. The general rule on the $(i+2)$ th mini-slot, $1 \leq i \leq M-1$, is that all active nodes with $b_{i}=0$ send RTBs; at the end of the mini-slot, if a COLLISION is alarmed, all active nodes with $b_{i}=1$ are backlogged and wait for the next slot; while a NULL is detected, all active nodes with $b_{i}=1$ remain active in the next mini-slot. This process continues running until a SINGLE is recognized. Clearly, at the end of the collision resolution process, only the active node with the lowest-numbered temporary_ID will be the winner. To ensure fairness, each node subtracts one $(\bmod N)$ from its current temporary_ID at the end of every slot. The advantage of the deterministic approach is that a winner is guaranteed to be elected if at least one active node exists. However, only the partial fairness can be achieved because of the multihop characteristic in ad-hoc networks. Besides, the value of $M$ by the deterministic approach may be larger than that by the randomized approach.

In packet transmission phase, every winner in every grid in the leader election phase starts to transmit. Since simultaneous reception of packets on other channels is not affected, all nodes can gain the data concurrently. The control packet length is typically smaller than the data packet length, it is worthwhile taking multiple mini-slots to compete for the access right. To sum up, our hybrid MAC protocol is similar to the leader election among active nodes within each gird in every slot.

\section{Performance Simulations}

Two bandwidth models have been proposed in [14] to evaluate the network throughput performance for multichannel ad-hoc networks. (i) Fixed-channelbandwidth: Each channel has a fixed bandwidth. The more the channels, the more bandwidth the network can potentially use. This model is especially suitable for CDMA environments. (ii) Fixed-total-bandwidth: The total bandwidth offered to the network is fixed. With more channels, each channel will have less bandwidth. This model is especially suitable for FDMA environments. 


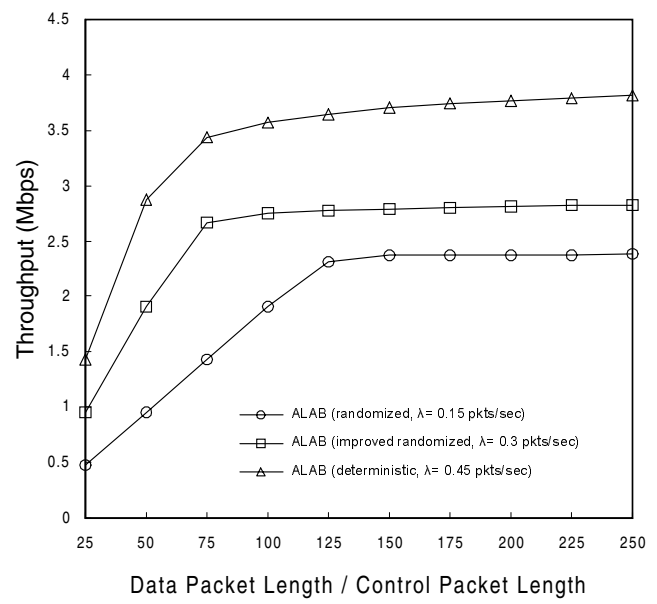

Fig. 3. $L_{d} / L_{c}$ versus throughput under the fixed-total-bandwidth model. $(\eta=8$ and $|\mathcal{G}|=10 \times 10$.)

Due to space limitations, mathematical analysis can be referred to our technical report 6]. In this section, we report the simulation results. We use the fixedincrement time advance approach [10] for our discrete-event simulation model to evaluate the performance of ALAB. We have developed a simulator by $\mathrm{C}++$. The ad-hoc network is simulated by placing $N$ nodes randomly and uniformly within a bounded geographic region. The geographic region size $\left(|\mathcal{G}|=\frac{\mathcal{A}}{d^{2}}\right)$ is measured by the number of grids. The transmission range of all simulated nodes is $r$ meters. The control packet length $L_{c}$ including the guard time is 20 bytes and the data packet length $L_{d}$ is a multiple of $L_{c}$. Network traffic was generated according to a Poisson arrival process with a mean of $\lambda$ packets per second, and uniformly distributed among the nodes. If the fixed-channel-bandwidth model is assumed, each channel's bandwidth is $1 \mathrm{Mbps}$. If the fixed-total-bandwidth is assumed, the total bandwidth is $1 \mathrm{Mbps}$. We will consider the effect of node density on the performance instead of the average degree, where the node density of the grid plane $\left(\eta=\frac{N}{|\mathcal{G}|}\right)$ is defined as the average number of nodes per grid.

A) Effect of Data Packet Length: In Fig. 3, we show the effect of the ratio $L_{d} / L_{c}$ on the throughput performance under the fixed-total-bandwidth model. In this experiment, we fix $\eta$ and $\mathcal{G}$ as 8 and $10 \times 10$, respectively. We can see that when $L_{d} / L_{c} \leq 125$, the throughput is highly promoted with the increasing length of data packet. This is because each successful leader election process can schedule more data bits to be sent. However, if we further increase the ratio $L_{d} / L_{c}$, the throughput of ALAB will be saturated at a certain point. As shown in Fig. 3. as both offered load and $L_{d} / L_{c}$ increase, the throughput of ALAB (deterministic collision resolution approach) approaches the network capacity.

B) Effect of Arrival Rate and Bandwidth Models: In this experiment, we assume that $N=512, r=2 d,|\mathcal{G}|=8 \times 8, \eta=8$, and $L_{d} / L_{c}=50$. Fig. 4 and 


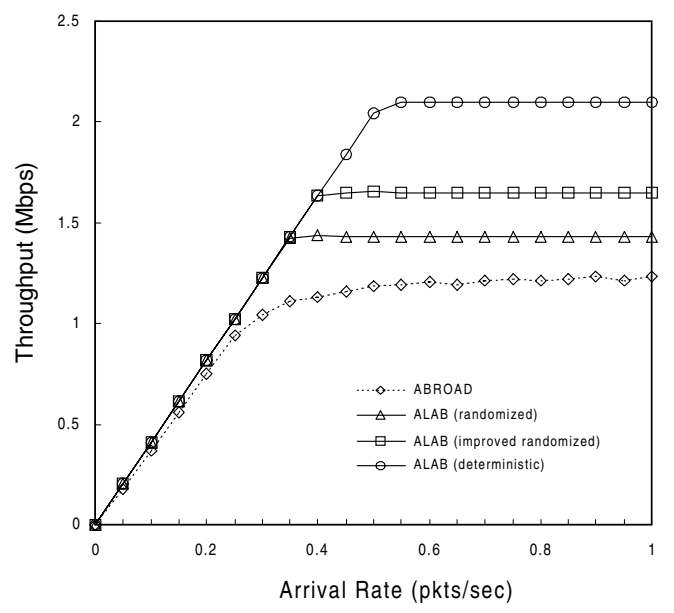

Fig. 4. Arrival rate versus throughput under the fixed-total-bandwidth model. $(N=$ $512, r=2 d, \eta=8,|\mathcal{G}|=8 \times 8$, and $L_{d} / L_{c}=50$.)

5] show the throughput versus the offered load under the fixed-total-bandwidth model and under the fixed-channel-bandwidth model respectively. Especially, even under the fixed-total-bandwidth model, we find a $70 \%$ increase in the peak performance for ALAB over ABROAD, which delivers superior performance than TDMA, IEEE 802.11, and ADAPT 45. The reasons are three-fold. (i) In ALAB, via the location-aware channel assignment scheme, the number of potential interfering terminals is significantly reduced from the size of two-hop neighborhood to the size of intra-grid neighborhood. (ii) Via the leader election process in ALAB, the probability for a node to reserve a slot is highly boosted. (iii) In such a crowed environment, the erasure effect [8] or deadlocks also cause the performance of ABROAD degradation. However, it is not very fair to compare ABROAD and ALAB because of their different assumptions on the transceivers. In Fig. 5, we see that the ALAB protocol with the deterministic collision resolution approach performs best since an active node is guaranteed to be elected (if it exists) in a grid in a slot.

C) Effect of Node Density: Fig. 6 shows the throughput versus node density and arrival rate under the fixed-channel-bandwidth model. We use $N=256$ and $L_{d} / L_{c}=75$. We see that as the node density decreases and/or the traffic load increases, the throughput increases monotonically and is finally saturated at a certain point. Especially, we find that when $\lambda=15 \sim 20$ and $\eta=4 \sim 16$, the deterministic collision resolution approach yields about $27.67 \% \sim 56.67 \%$ improvement in the throughput, as compared with the randomized one. This is reasonable due to the uncertainty in the leader election phase by the randomized approach. Given fixed $\mathcal{A}$ and $N$, decreasing the node density will promote the throughput; meanwhile, it will cause the number of grids increase. Since we restrict $\sqrt{2} d \leq r \leq 2 d$ in our design, a larger number of grids implies a shorter 


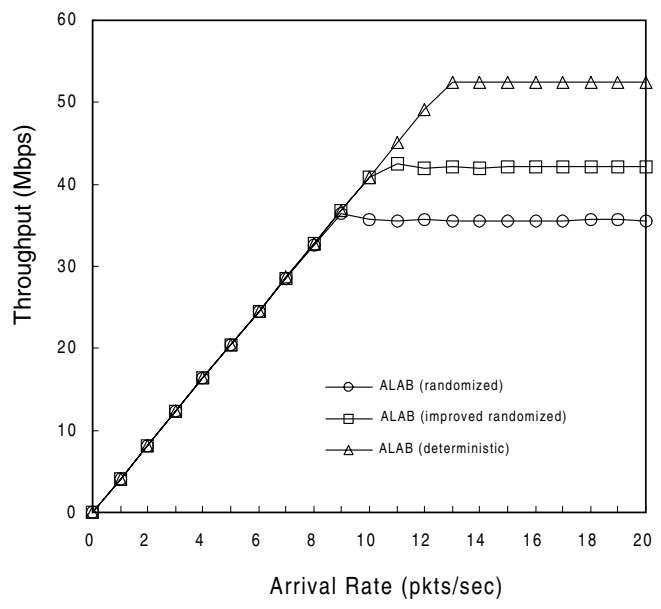

Fig. 5. Arrival rate versus throughput under the fixed-channel-bandwidth model. $(N=$ $512, \eta=8,|\mathcal{G}|=8 \times 8$, and $L_{d} / L_{c}=50$.)

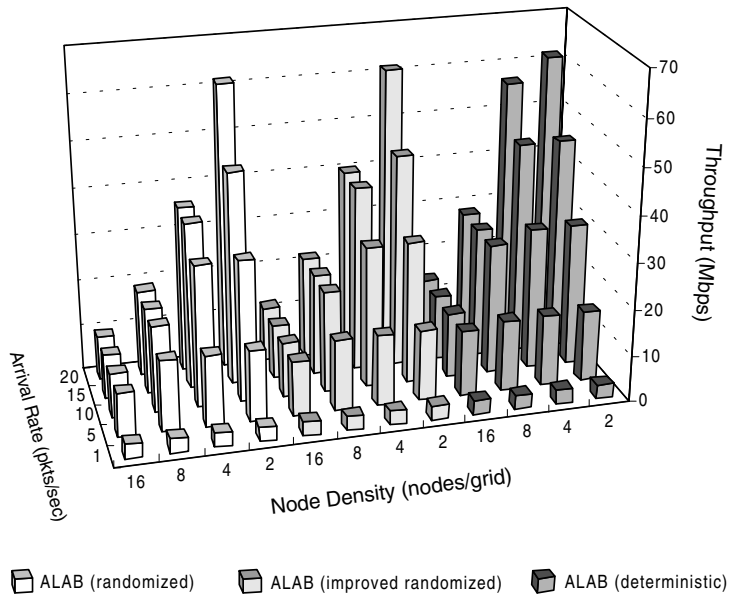

Fig. 6. Throughput versus node density and arrival rate under the fixed-channelbandwidth model. $\left(N=256\right.$ and $L_{d} / L_{c}=75$.)

transmission range. From the perspective of the routing performance, this will result in more hops from sources to destinations. To sum up, determining the optimal values of $r$ and $d$ is not an easy task.

D) Effect of Node ID Distribution: In all the above experiments, we have observed that the ALAB protocol with the deterministic collision resolution approach performs best. However, its collision resolution method highly depends on the distribution of the node IDs. In spite of the multihop characteristic in ad-hoc networks, each contending station should receive an equal share of the 
transmission bandwidth. We conduct an experiment to understand this fairness issue. We use $N=16, \eta=4,|\mathcal{G}|=4$, and $L_{d} / L_{c}=75$. Four sample nodes intended for our observation are 0000, 0001, 1010, and 1011. Furthermore, we assume that they are located in a same grid. Fig. 7 shows the simulation result under the fixed-channel-bandwidth model. We see that as the offered load increases, the performance range of the sample nodes increases significantly. That is, the unfairness problem becomes serious when traffic load is heavy. Therefore, if fairness is critical, the ALAB protocol with the improved randomized collision resolution approach may be a compromise solution.

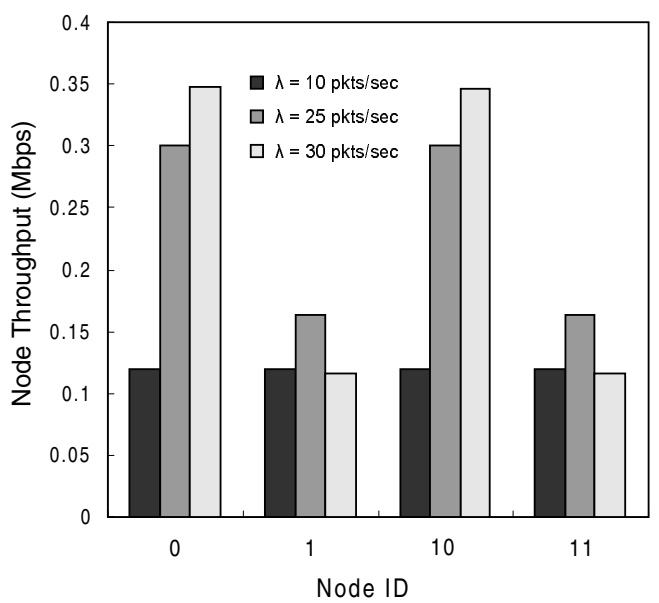

Fig. 7. Node ID versus node throughput under the fixed-channel-bandwidth model. $\left(N=16, \eta=4,|\mathcal{G}|=4\right.$, and $L_{d} / L_{c}=75$.)

\section{Conclusions}

In this paper, we have proposed a new adaptive location-aware MAC protocol, called ALAB, for link-level broadcast support in multichannel MANETs. By virtue of GPS and channel assign scheme, all nodes within the same grid are able to maintain a consistent channel view. Due to the channel consistency in every grid, no deadlock or hidden terminal problems will exist. ALAB is scalable and mobility-transparent since it does not require any link state information. Using the ternary channel feedback information, our novel hybrid broadcast scheme can achieve high throughput performance. In principle, ALAB tries to combine both of the advantages of the allocation- and contention-based protocols and overcomes their individual drawbacks. ALAB has deterministic access guarantees by its base TDMA allocation protocol while providing flexible and efficient bandwidth management by reclaiming unused slots through the stable 
tree-splitting algorithms. Extensive experimental results have been conducted, which take many factors, such as channel bandwidth models, arrival rate, data packet length, node density, and fairness, into consideration. Both analysis [6] and simulation results do confirm the advantage of our scheme over other MAC protocols, such as IEEE 802.11, ADAPT [4], and ABROAD [5], even under the fixed-total-bandwidth model. All these results make ALAB a promising protocol to enhance the performance of the MANET.

\section{References}

1. G. Anastasi, L. Lenzini, and E. Mingozzi. HIPERLAN/1 MAC protocol: Stability and performance analysis. IEEE Journal on Selected Areas in Communications, Vol. 18, No. 9, Sep., (2000) 1787-1798.

2. D. Bertsekas and R. Gallager. Data Networks, Second Edition, Prentice-Hall, 1992.

3. I. Chlamtac and A. Faragó. An optimal channel access protocol with multiple reception capacity. IEEE Trans. on Computers, Vol. 43, No. 4, (1994) 480-484.

4. I. Chlamtac, A. Faragó, A. D. Myers, V. R. Syrotiuk, and G. Záruba. ADAPT: a dynamically self-adjusting media access control protocol for ad hoc networks. GLOBECOM '99, Vol. 1A, (1999) 11-15.

5. I. Chlamtac, A. D. Myers, V. R. Syrotiuk, and G. Záruba. An adaptive medium access control (MAC) protocol for reliable broadcast in wireless networks. IEEE International Conference on Communications, Vol. 3, (2000) 1692-1696.

6. Z.-T. Chou, C.-C. Hsu, and F.-C. Lin. An adaptive location-aware MAC Protocol for multichannel multihop ad-hoc networks. Technical Report, National Taiwan University, 2001.

7. R. Garcés and J.J. Garcia-Luna-Aceves. Collision avoidance and resolution multiple access with transmission queues. Wireless Networks, Vol. 5, (1999) 95-109.

8. T.-C. Hou and T.-J. Tsai. An access-based clustering protocol for multihop wireless ad hoc networks. IEEE Journal on Selected Areas in Communications, Vol. 19, No. 7, July, (2001) 1201-1210.

9. J.-H. Ju and V. O. K. Li. TDMA scheduling design of multihop packet radio networks based on Latin squares. IEEE Journal on Selected Areas in Communications, Vol. 7, No. 8, Aug., (1999) 1345-1352.

10. Averill M. Law and W. David Kelton. Simulation Modeling and Analysis, Third Edition, McGrraw-Hill Book Company Inc., 2000.

11. M. K. Marina, G. D. Kondylis, and U. C. Kozat. RBRP: A robust broadcast reservation protocol for mobile ad hoc networks. IEEE International Conference on Communications, Vol. 3, (2001) 878-885.

12. K. Nakano and S. Olariu. Randomized initialization protocols for ad hoc networks. IEEE Trans. Parallel and Distributed Systems, Vol. 11, No. 7, July, (2000) 749-759.

13. Z. Tang and J.J. Garcia-Luna-Aceves. A protocol for topology-dependent transmission scheduling in wireless networks. IEEE Wireless Communications and Networking Conference, Vol. 3, (1999) 1333-1337.

14. Y.-C. Tseng, S.-L. Wu, C.-M. Chao, and J.-P. Sheu. Location-aware channel assignment for a multi-channel mobile ad hoc network. ICS2000 Workshop on Computer Networks, Internet, and Multimedia, 2000.

15. C. Zhu and M. Corson. A five-phase reservation protocol (FPRP) for mobile ad hoc networks. Wireless Networks, Vol. 7, (2001) 371-384. 